\title{
Antibiotic susceptibility pattern of Klebsiella pneumoniae isolated from sputum, urine and pus samples
}

\author{
${ }^{1}$ Dr.R.Sarathbabu, ${ }^{2}$ Dr.T.V.Ramani, ${ }^{3}$ Dr. K.Bhaskara rao, ${ }^{4}$ Dr.Supriya Panda \\ ${ }^{1}$ M.D., Assistant Professor, M.D., ${ }^{2}$ Professor and Head., ${ }^{3}$ M.D., Professor., ${ }^{4}$ M.D., Professor. \\ Department of Microbiology, Maharajah's institute of medical sciences, Nellimarla, Vizianagaram, \\ Andhrapradesh, India.
}

\begin{abstract}
Introduction: Gram-negative pathogens are an important cause of hospital acquired infections throughout the world. Klebsiella pneumoniae has become one of the more common causes of nosocomial infections.

Materials and methods: A total number of 1264 urine, 544 pus and 784 sputum samples from January 2008 to October 2010 were included in the study. Isolates of klebsiella pneumoniae were identified by their morphological and biochemical characteristics. All the isolates of klebsiella pneumoniae identified were subjected to antibiotic sensitivity testing by modified Kirby-bauer disc diffusion method.

Results: The number of klebsiella pneumoniae isolates were 254 from 1264 urine samples, 135 from 544 pus samples and 191 from 784 sputum samples. Majority of the strains isolated were sensitive to Amikacin.

Conclusion: The present study from 2008 to 2010 reveals the incidence of infections due to klebsiella pneumoniae strains in the hospitalized patients and their tendency towards antibiotic resistance.

Key words: Kebsiella pneumoniae, nosocomial infections, pneumonia, urinary tract infections, beta-lactamase, ESBL's, Gram-negative, non-motile, urease.

\section{Introduction}

Klebsiella pneumoniae is a Gram-negative, non-motile, encapsulated, lactose fermenting, facultative anaerobic, rod shaped bacterium found in the normal flora of the mouth, skin and intestines.In the recent years, klebsiella pneumoniae has become important pathogen in nosocomial infections. Klebsiella pneumoniae is most frequently recovered from clinical specimens and can cause a classic form of primary pneumonia. Klebsiella pneumoniae can also cause a variety of extrapulmonary infections, including enteritis and meningitis in infants, urinary tract infections in children and adults and septicaemia. In the United States, klebsiella accounts for 3-7\% of all nosocomial bacterial infections, placing them among the eight most important infectious pathogens in hospitals. Klebsiellae have a tendency to harbor antibiotic resistant plasmids; thus, infections with multiple antibiotic-resistant strains can be anticipated.

Virtually all clinical strains are resistant to ampicillin, carbenicillin, and ticarcillin. Of particular concern is the recent appearance of klebsiella strains that possess plasmids that mediate resistance to ESBL drugs. This form of resistance is due to the production of unique beta-lactamase enzymes, referred to as ESBL's. These enzymes have been seen mostly in strains of klebsiella pneumoniae and E.coli, and cause them to be resistant to most beta-lactam drugs, including the third generation cephalosporins.
\end{abstract}

Aim of the present study was to know the antibiotic sensitivity pattern of klebsiella pneumoniae strains isolated from sputum, urine and pus samples sent to Microbiology laboratory from 2008 to 2010.

\section{Materials and methods}

Sputum, urine, and pus samples collected from inpatients admitted into clinical wards were sent to Microbiology laboratory. The samples were inoculated on blood agar and mac conkey agar and incubated overnight at $37^{\circ} \mathrm{C}$. Klebsiella pneumoniae strains were identified by their morphology and biochemical characteristics. Morphology of klebsiella pneumoniae identified were large, dome-shaped, mucoid colonies on blood agar and lactose fermenting colonies on mac conkey agar. In Gram-staining, gram-negative, short, plump, straight rods were seen. The biochemical characters identified were negative indole test, negative methyl red test, positive voges-proskauer test, positive citrate utilization test, positive urease test, acid and abundant gas production from glucose, lactose, sucrose, maltose and mannitol sugar fermentation tests.

Antibiotic sensitivity testing was done for all the isolates on Mueller-hinton agar by modified Kirby-bauer disc diffusion technique.The following antibiotic discs were used for testing bearing the concentrations:

Pus and sputum samples: 
1.Amoxyclav - $10 \mathrm{mcg}$.

2.Amikacin - 30mcg.

3.Ampicillin+Sulbactum - $10 \mathrm{mcg} / 10 \mathrm{mcg}$

4.Cefotaxime - 30mcg.

5.Ceftriaxone $-30 \mathrm{mcg}$.

6.Gentamicin - $10 \mathrm{mcg}$.

7.Ofloxacin - 5mcg.

8.Netilmycin - 30mcg.

\section{Urine samples:}

1.Ampicillin+Sulbactam $-10 \mathrm{mcg} / 10 \mathrm{mcg}$.

2.Amikacin $-30 \mathrm{mcg}$.

3.Co-trimoxazole $-25 \mathrm{mcg}$.

4.Cefazolin $-30 \mathrm{mcg}$.

5.Ceftriaxone $-30 \mathrm{mcg}$.

6.Gentamicin $-10 \mathrm{mcg}$.

7.Nitrofuratoin $-300 \mathrm{mcg}$.

8.Ofloxacin $-5 \mathrm{mcg}$.

9. Tetracycline $-30 \mathrm{mcg}$.
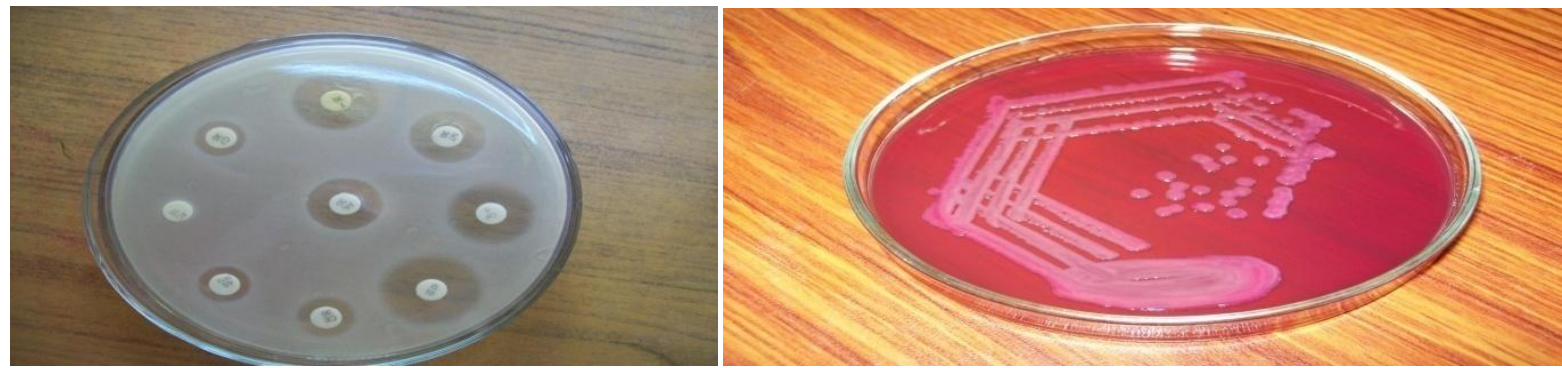

\section{Results}

Table - 1.

Total no.of samples:

\begin{tabular}{|l|l|l|l|l|}
\hline Sample & 2008 & 2009 & 2010 & Total \\
\hline Sputum & 267 & 297 & 220 & 784 \\
\hline Pus & 180 & 191 & 173 & 544 \\
\hline Urine & 386 & 486 & 392 & 1264 \\
\hline
\end{tabular}

Table - 2

Culture positivity of Klebsiella pneumoniae:

\begin{tabular}{|l|l|l|l|}
\hline Sample & 2008 & 2009 & 2010 \\
\hline Sputum & $\mathrm{N}=54(20.22 \%)$ & $\mathrm{N}=72(24.24 \%)$ & $\mathrm{N}=65(29.55 \%)$ \\
\hline Urine & $\mathrm{N}=83(21.5 \%)$ & $\mathrm{N}=90(18.52 \%)$ & $\mathrm{N}=81(20.66 \%)$ \\
\hline Pus & $\mathrm{N}=45(25 \%)$ & $\mathrm{N}=54(28.27 \%)$ & $\mathrm{N}=36(20.81 \%)$ \\
\hline
\end{tabular}


Table - 3:

Antibiogram of klebsiella pneumoniae isolated from pus samples:

\begin{tabular}{|c|c|c|c|c|c|c|c|c|c|c|c|c|}
\hline \multirow[t]{3}{*}{ NAME } & \multicolumn{4}{|c|}{$2008(\mathrm{~N}=45)$} & \multicolumn{4}{|c|}{$2009(\mathrm{~N}=54)$} & \multicolumn{4}{|c|}{$2010(\mathrm{~N}=36)$} \\
\hline & \multicolumn{2}{|c|}{ SENSITIVE } & \multicolumn{2}{|c|}{ RESISTANT } & \multicolumn{2}{|c|}{ SENSITIVE } & \multicolumn{2}{|c|}{$\begin{array}{l}\text { RESISTAN } \\
\mathrm{T}\end{array}$} & \multicolumn{2}{|c|}{ SENSITIVE } & \multicolumn{2}{|c|}{ RESISTANT } \\
\hline & NO & $\%$ & $\mathrm{NO}$ & $\%$ & NO & $\%$ & NO & $\%$ & NO & $\%$ & $\mathrm{NO}$ & $\%$ \\
\hline AMOXYCLAV & 29 & $\begin{array}{l}64 . \\
44\end{array}$ & 16 & $\begin{array}{l}35 . \\
56\end{array}$ & 32 & $\begin{array}{l}59 . \\
26\end{array}$ & 22 & $\begin{array}{l}40 . \\
74\end{array}$ & 20 & $\begin{array}{l}55.5 \\
5\end{array}$ & 16 & $\begin{array}{l}44.4 \\
5\end{array}$ \\
\hline CEFOT AXIME & 24 & $\begin{array}{l}53 . \\
33\end{array}$ & 21 & $\begin{array}{l}46 . \\
67\end{array}$ & 29 & $\begin{array}{l}53 . \\
71\end{array}$ & 25 & $\begin{array}{l}46 . \\
29\end{array}$ & 17 & $\begin{array}{l}47.2 \\
2\end{array}$ & 19 & $\begin{array}{l}52.7 \\
8\end{array}$ \\
\hline AMIKACIN & 34 & $\begin{array}{l}75 . \\
56\end{array}$ & 11 & $\begin{array}{l}24 . \\
44\end{array}$ & 38 & $\begin{array}{l}70 . \\
37\end{array}$ & 16 & $\begin{array}{l}29 . \\
63\end{array}$ & 24 & $\begin{array}{l}66.6 \\
7\end{array}$ & 12 & $\begin{array}{l}33.3 \\
3\end{array}$ \\
\hline GENTAMICIN & 30 & $\begin{array}{l}66 . \\
67\end{array}$ & 15 & $\begin{array}{l}33 . \\
33\end{array}$ & 32 & $\begin{array}{l}59 . \\
23\end{array}$ & 22 & $\begin{array}{l}40 . \\
74\end{array}$ & 21 & $\begin{array}{l}58.3 \\
3\end{array}$ & 15 & $\begin{array}{l}41.6 \\
7\end{array}$ \\
\hline $\begin{array}{l}\text { AMPICILLIN+SULB } \\
\text { ACTUM }\end{array}$ & 26 & $\begin{array}{l}57 . \\
78\end{array}$ & 19 & $\begin{array}{l}42 . \\
22\end{array}$ & 30 & $\begin{array}{l}55 . \\
55\end{array}$ & 24 & $\begin{array}{l}44 . \\
44\end{array}$ & 19 & $\begin{array}{l}52.7 \\
8\end{array}$ & 17 & $\begin{array}{l}47.2 \\
2\end{array}$ \\
\hline OFLOXACIN & 19 & $\begin{array}{l}42 . \\
22\end{array}$ & 26 & $\begin{array}{l}57 . \\
78\end{array}$ & 28 & $\begin{array}{l}51 . \\
85\end{array}$ & 26 & $\begin{array}{l}48 . \\
15\end{array}$ & 17 & $\begin{array}{l}47.2 \\
2\end{array}$ & 19 & $\begin{array}{l}52.7 \\
8\end{array}$ \\
\hline TETRACYCLINE & 20 & $\begin{array}{l}44 . \\
45\end{array}$ & 25 & $\begin{array}{l}55 . \\
56\end{array}$ & 23 & $\begin{array}{l}42 . \\
59\end{array}$ & 31 & $\begin{array}{l}57 . \\
41\end{array}$ & 15 & $\begin{array}{l}41.6 \\
7\end{array}$ & 21 & $\begin{array}{l}58.3 \\
3\end{array}$ \\
\hline NETILMICIN & 26 & $\begin{array}{l}57 . \\
78\end{array}$ & 19 & $\begin{array}{l}42 . \\
22\end{array}$ & 31 & $\begin{array}{l}57 . \\
41\end{array}$ & 23 & $\begin{array}{l}42 . \\
59\end{array}$ & 22 & $\begin{array}{l}61.1 \\
1\end{array}$ & 14 & $\begin{array}{l}38.8 \\
9\end{array}$ \\
\hline CEFTRIAXONE & 23 & $\begin{array}{l}51 . \\
11\end{array}$ & 22 & $\begin{array}{l}48 . \\
89\end{array}$ & 28 & $\begin{array}{l}51 . \\
85\end{array}$ & 26 & $\begin{array}{l}48 . \\
15\end{array}$ & 15 & $\begin{array}{l}41.6 \\
7\end{array}$ & 21 & $\begin{array}{l}58.3 \\
3\end{array}$ \\
\hline
\end{tabular}


Table - 4:

Antibiogram of klebsiella pneumoniae isolated from sputum samples:

\begin{tabular}{|c|c|c|c|c|c|c|c|c|c|c|c|c|}
\hline \multirow[t]{3}{*}{ NAME } & \multicolumn{4}{|c|}{$2008(\mathrm{~N}=54)$} & \multicolumn{4}{|c|}{$2009(\mathrm{~N}=72)$} & \multicolumn{4}{|c|}{$2010(\mathrm{~N}=65)$} \\
\hline & \multicolumn{2}{|c|}{ SENSITIVE } & \multicolumn{2}{|c|}{ RESISTANT } & \multicolumn{2}{|c|}{ SENSITIVE } & \multicolumn{2}{|c|}{ RESISTANT } & \multicolumn{2}{|c|}{ SENSITIVE } & \multicolumn{2}{|c|}{$\begin{array}{l}\text { RESISTAN } \\
\mathrm{T}\end{array}$} \\
\hline & NO & $\%$ & NO & $\%$ & NO & $\%$ & NO & $\%$ & NO & $\%$ & NO & $\%$ \\
\hline AMOXYCLAV & 33 & 61.11 & 21 & 38.89 & 42 & 58.33 & 30 & 41.67 & 35 & 53.85 & 30 & $\begin{array}{l}46 . \\
15\end{array}$ \\
\hline CEFOTAXIME & 28 & 51.85 & 26 & 48.15 & 38 & 52.77 & 34 & 47.23 & 31 & 47.69 & 34 & $\begin{array}{l}52 . \\
31\end{array}$ \\
\hline AMIKACIN & 36 & 66.67 & 18 & 33.33 & 44 & 61.11 & 28 & 38.88 & 37 & 56.92 & 28 & $\begin{array}{l}43 . \\
08\end{array}$ \\
\hline GENTAMICIN & 28 & 51.85 & 26 & 48.15 & 34 & 47.23 & 38 & 52.77 & 30 & 46.15 & 35 & $\begin{array}{l}53 . \\
85\end{array}$ \\
\hline Ampicillin+Sulbactum & 30 & 55.55 & 24 & 44.45 & 39 & 54.17 & 33 & 45.83 & 34 & 52.31 & 31 & $\begin{array}{l}47 . \\
69\end{array}$ \\
\hline OFLOXACIN & 27 & 50 & 27 & 50 & 38 & 52.77 & 34 & 47.23 & 30 & 46.15 & 35 & $\begin{array}{l}53 . \\
85\end{array}$ \\
\hline TETRACYCLINE & 26 & 48.15 & 28 & 51.85 & 33 & 45.83 & 39 & 54.17 & 26 & 40 & 39 & 60 \\
\hline NETILMYCIN & 34 & 62.96 & 20 & 37.04 & 43 & 59.72 & 29 & 40.28 & 39 & 60 & 26 & 40 \\
\hline CEFTRIAXONE & 26 & 48.15 & 28 & 51.85 & 33 & 45.83 & 39 & 54.17 & 27 & 41.54 & 38 & $\begin{array}{l}58 . \\
46\end{array}$ \\
\hline
\end{tabular}


$\underline{\text { Table - 5: }}$

Antibiogram of klebsiella pneumoniae isolated from urine samples:

\begin{tabular}{|c|c|c|c|c|c|c|c|c|c|c|c|c|}
\hline \multirow[t]{3}{*}{ Name. } & \multicolumn{4}{|c|}{$2008(\mathrm{~N}=83)$} & \multicolumn{4}{|c|}{$2009(\mathrm{~N}=90)$} & \multicolumn{4}{|c|}{$2010(\mathrm{~N}=81)$} \\
\hline & \multicolumn{2}{|c|}{ SENSITIVE } & \multicolumn{2}{|c|}{ RESISTANT } & \multicolumn{2}{|c|}{ SENSITIVE } & \multicolumn{2}{|c|}{ RESISTANT } & \multicolumn{2}{|c|}{ SENSITIVE } & \multicolumn{2}{|c|}{ RESISTANT } \\
\hline & NO & $\%$ & NO & $\%$ & NO & $\%$ & NO & $\%$ & NO & $\%$ & NO & $\%$ \\
\hline
\end{tabular}

\begin{tabular}{|l|l|l|l|l|l|l|l|l|l|l|l|l|}
\hline NITROFURATOIN & 42 & 50.60 & 41 & 49.39 & 43 & 47.78 & 47 & 52.22 & 38 & 46.92 & 43 & 53.08 \\
\hline Co-trimoxazole & 41 & 49.39 & 42 & 50.60 & 42 & 46.67 & 48 & 53.33 & 35 & 43.21 & 46 & 56.79 \\
\hline OFLOXACIN & 46 & 55.42 & 37 & 44.57 & 45 & 50 & 45 & 50 & 38 & 46.92 & 43 & 53.08 \\
\hline GENTAMICIN & 51 & 61.45 & 32 & 38.55 & 52 & 57.78 & 38 & 42.22 & 42 & 51.85 & 39 & 48.15 \\
\hline AMIKACIN & $\mathbf{6 5}$ & $\mathbf{7 8 . 3 1}$ & $\mathbf{1 8}$ & $\mathbf{2 1 . 6 9}$ & $\mathbf{6 7}$ & $\mathbf{7 4 . 4 4}$ & $\mathbf{2 3}$ & $\mathbf{2 5 . 5 6}$ & $\mathbf{5 8}$ & $\mathbf{7 1 . 6 0}$ & $\mathbf{2 3}$ & $\mathbf{2 8 . 4 0}$ \\
\hline CEFAZOLIN & 48 & 57.83 & 35 & 42.17 & 41 & 45.56 & 49 & 54.44 & 35 & 43.21 & 46 & 56.79 \\
\hline Ampicillin+Sulbactam & 54 & 65.06 & 29 & 34.94 & 55 & 61.11 & 35 & 38.89 & 48 & 59.23 & 33 & 40.74 \\
\hline CEFTRIAXONE & 44 & 53.01 & 39 & 46.98 & 43 & 47.78 & 47 & 52.22 & 37 & 45.67 & 44 & 54.33 \\
\hline
\end{tabular}

\section{Discussion}

Klebsiella pneumonia is most frequently recovered from clinical specimens and can cause a classic form of primary pneumonia.It is infrequently found in the oropharynx of normal persons $(1-6 \%$ carrier rate).However, a prevalence rate as high as $20 \%$ may occur in hospitalized patients. The higher incidence of infections due to klebsiella pneumonia during the past decade probably reflects both an increase in nosocomial infections in the debilitated or immunosuppressed individuals and a trend towards greater antibiotic resistance. In the present study from 2008 to 2010 , culture positivity for klebsiella pneumoniae was $24.36 \%$ for sputum samples, $20.09 \%$ for urine samples and $24.82 \%$ for pus samples.I

In the present study, majority of the strains islated were sensitive to amikacin ( $\mathrm{P}$ Value $=0.50$ ). The percentage of sensitivity to amikacin was $\mathbf{7 5 . 5 6 \%}$ in 2008 , 70.37\% in 2009 , and $\mathbf{6 6 . 6 7 \%}$ in 2010 for pus samples; $\mathbf{6 6 . 6 7 \%}$ in 2008, 61.11\% in 2009 and $\mathbf{5 6 . 9 2 \%}$ in 2010 for sputum samples and $\mathbf{7 8 . 3 1 \%}$ in 2008 , $\mathbf{7 4 . 4 4 \%}$ in 2009 and $\mathbf{7 1 . 6 0 \%}$ in 2010 for urine samples; Thus showing a gradual increase in resistance and decrease in sensitivity.

\section{Conclusion}

The present study from 2008 to 2010 reveals the incidence of infections due to klebsiella pneumoniae strains in the hospitalized patients and their tendency towards antibiotic resistance. Majority of the strains isolated were sensitive to amikacin. A gradual increase in resistance and decrease in sensitivity was observed for all the drugs tested towards klebsiella pneumoniae strains isolated from sputum, urine and pus samples from 2008 to 2010 . 


\section{Referrences:}

[1] 1.Hadi Mehrgan, Mohammad Rahbar, Zohreh Arab-Halvaii, "High prevalence of extended spectrum beta lactamase producing Klebsiella pneumoniae in a tertiary care hospital in Tehran, Iran", The Journal of infection in developing countries,Vol.4,No.3,2009.

[2] 2.M.Shahid, A.Malik, M.Akram, L.M.Agarwal, A.U.Khan, M.Agarwal, "Prevalent phenotypes and antibiotic resistance in Escherichia coli and klebsiella pneumonia at an Indian tertiary care hospital: plasmid-mediated cefoxitin resistance", International journal of infectious diseases, Vol.2, issue 3, May 2008, Pg:256-264.

[3] 3.Aminzadeh.Z, Sadat kashi.M, Shabani.M, "Bacteriuria by extended spectrum beta lactamase producing Escherichia coli and klebsiella pneumonia isolates in a government hospital in south of Tehran, Iran, Iranian journal of kidney diseases, 2008 Oct;2(4):197-200

[4] 4.Ami Y Varaiya, Jyotsana D Dogra, Manasi H Kulkarni, Pallavi N Bhalekar, "Extended spectrum beta lactamase producing Escherichia coli and klebsiella pneumonia in diabetic foot infections", Indian journal of medical microbiology, Vol.51, issue 3, 2008, Pg:370-372.

[5] 5. Anguzyjr.R, Olila.D, "Drug sensitivity patterns of bacterial isolates from septic post-operative wounds in a regional referral hospital in Uganda", Journal of African health sciences, 2007 Sep:7(3):Pg:148-54.

[6] 6.Yengkokpam.C, Inqudam.D, Yengkokpam.I.S., Jha.BK, “Antibiotic susceptibility pattern of urinary isolates in Imphal(Manipur), India”, Nepal medical college journal, 2007Sep;9(3):170-2.

[7] 7.Shobha KL, Gowrish rao.S, Sugandhi rao, Sreeja C.K., "Prevalence of extended spectrum beta lactamases in urinary isolates of Escherichia coli, Klebsiella and Citrobacter species and their antimicrobial susceptibility pattern in a tertiary care hospital," Indian journal for the Practising Doctor, Vol.3,No.6(2007-01-2007-02).

[8] 8.Krista loivukene,Epp Sepp, Vivika Adamson, Piret Mitt, Ulle Kallandi, Karin Otter, Paul Naaber, "Prevalence and antibiotic susceptibility of Acinetobacter baumanni, Pseudomonas aeruginosa and Klebsiella pneumonia in Estonian intensive care units in comparison with European Data", Scandinavian journal of infectious diseases, 2006, Vol.38,No.11-12,Pg:1001-1008

[9] 9.Das RN, Chandrasekhar TS, Joshi HS, Gurung M, Shrestha N, Shivananda PG, "Frequency and susceptibility profile of pathogens causing urinary tract infections at a tertiary care hospital in Western Nepal," Singapore medical journal, 2006;47(4):281.

[10] 10.Arya M, Arya PK, Biswas D, Prasad R, "Antimicrobial susceptibility pattern of bacterial isolates from post-operative wound infections", Indian journal of Pathology and Microbiology, 2005 Apr 48(2):266-9.

[11] 11.Kader AA, kumar A, "Prevalence and antimicrobial susceptibility of extended spectrum beta lactamase producing Escherichia coli and Klebsiella pneumoniae in a general hospital",2005 May-Jun 25(3);239-42.

[12] 12.Seid J, Asrat D, "Occurrence of extended spectrum beta lactamase enzymes in clinical isolates of Klebsiella species from Harar region, Eastern Ethiopia," Acta Tropica Journal, 2005 Aug:95(2):143-8.

[13] 13.J Bishara, G livne, S.Ashkenazi, S.Pitlik, "Antimicrobial susceptibility of extended spectrum beta lactamase producing Klebsiella pneumoniae and Escherichia coli”, Israel medical association journal, 2005,Vol(7):298 -301.

[14] 14.CN Akujobi, "Antimicrobial susceptibility pattern of Klebsiella species from Ebonyi State University Teaching Hospital, Abakaliki,Nigeria”, Nigerian journal of Clinical Practice, Vol.8(2)2005:90-93

[15] 15.Grover SS, Sharma M, Pasha ST, Singh G, Lal S, "Antimicrobial susceptibility pattern and prevalence of extended spectrum beta lactamases producing strains of Klebsiella pneumonia from a major hospital in New Delhi”, Journal of Communicable diseases, 2004 Mar 36(1);17-26.

[16] 16.Z.Daoud, N.Hakime, "Prevalence and susceptibility patterns of extended spectrum beta lactamase producing Escherichia coli and Klebsiella pneumonaie in a general university hospital in Bierut, Lebanon”, 2003;Vol.16,No.2,Pg:233-238, Sociedad Espanola de Quimioterapia.

[17] 17.A.Subha, S.Ananthan, "Extended spectrum beta lactamase resistance to third generation cephalosporins among Klebsiella pneumonia in Chennai", Indian journal of medical Microbiology", 2002,Vol.20.issue 2 Pg:92-95.

[18] 18.Ingo Stock, Bernd Wiedemann, "Natural antibiotic susceptibility of Klebsiella pneumonia, K.oxytoca, K.panticola, K.ornitholytica and K.terrigena strains", Journal of Medical Microbiology, 2001, Vol.50, No.5,396-406.

[19] 19.Misra R, Kumar M, Menon PK, Ohri VC, "Plasmid mediated antibiotic resistance in Klebsiella pneumoniae", Indian journal of Pathology and Microbiology, 2001 Oct:44(4):427-9.

[20] 20.Kawakami S, Ono Y, Yamamoto M, Matumura M, Okamoto R, Inoue M, Miyazawa Y, "Extended spectrum beta lactamase produced by Escherichia coli and Klebsiella pneumoniae isolated from Teikyou university hospital-the first report", Journal of Japanese infectious and communicable diseases,1999 Nov:73(11):110-5. 